\title{
Recent results of the NA48/2 experiment
}

\author{
M. Raggi \\ Laboratori Nazionali di Frascati, Via E. Fermi 40, 00147 \\ Frascati, Italy
}

\begin{abstract}
NA48/2 collected the wolrd largest sample of $K^{ \pm} \rightarrow \pi^{ \pm} \pi^{0} \gamma$ decays. Direct Emission (DE) and Interference (INT) fractions with respect to the internal bremsstrahlung (IB) have been measured in the range $0<T_{\pi}^{*}<80 \mathrm{MeV}$ :

$\operatorname{Frac}_{\mathrm{DE}}\left(0<T_{\pi}^{*}<80 \mathrm{MeV}\right)=\left(3.32 \pm 0.15_{\text {sta }} \pm 0.14_{\text {sys }}\right) \times 10^{-2}$

$\operatorname{Frac}_{\mathrm{INT}}\left(0<T_{\pi}^{*}<80 \mathrm{MeV}\right)=-\left(2.35 \pm 0.35_{\text {sta }} \pm 0.39_{\text {sys }}\right) \times 10^{-2}$

where $T_{\pi}^{*}$ is the kinetic energy of the charged pion in the kaon rest frame.

A sample of $7253 K^{ \pm} \rightarrow \pi^{ \pm} e^{+} e^{-}$decay candidates has been collected by the NA48/2 experiment. The branching ratio in the full kinematic range was measured to be $B R=$ $(3.11 \pm 0.12) \cdot 10^{-7}$. For both decays CP violating asymmetry has been studied.
\end{abstract}

\section{Introduction}

The NA48/2 experiment at CERN SPS has collected the world largest amount of charged ka decays. During two runs in 2003 and 2004, about $18 \cdot 10^{9}$ events were recorded. The main go of NA48/2 was the search for direct CP violation in $K^{ \pm}$decays into three pions. Howeve given the high statistics achieved, many tests of Chiral Perturbation Theory (ChPT) were al possible in rare Kaon decays. In the following sections, recent tests of ChPT performed by $\mathrm{t}$ NA48/2 collaboration using $K^{ \pm} \rightarrow \pi^{ \pm} \pi^{0} \gamma$ and $K^{ \pm} \rightarrow \pi^{ \pm} e^{+} e^{-}$decays will be presented.

\section{$1 \quad$ Study of $K^{ \pm} \rightarrow \pi^{ \pm} \pi^{0} \gamma$ decay}

The total amplitude of the $K^{ \pm} \rightarrow \pi^{ \pm} \pi^{0} \gamma$ decay is the sum of two terms: the inner bremsstrahlu associated with the $K^{ \pm} \rightarrow \pi^{ \pm} \pi^{0}$ decay with a photon emitted from the outgoing charged pio and the direct emission in which the photon is emitted at the weak vertex. Using the Low th orem the branching ratio of the IB component can be predicted from that of the $K^{ \pm} \rightarrow \pi^{ \pm}$ channel, using QED corrections ${ }^{2,3}$.

The DE term has been extensively studied in the framework of Chiral Perturbation Theo $(\mathrm{ChPT})^{4,5,6}$. Direct photon emission can occur through both electric and magnetic dipc transitions. The electric dipole transition can interfere with the IB amplitude giving rise an interference term, which can have $\mathrm{CP}$ violating contributions. The magnetic part at ord $O\left(p^{4}\right)$ in ChPT is the sum of two anomalous amplitudes: one reducible, that can be calculat using the Wess-Zumino-Witten functional, and one direct amplitude, whose size is not mod independent but is expected to be small. There is no definite prediction from ChPT for $t$ electric transition amplitude, which depends on undetermined constants.

The properties of the $K^{ \pm} \rightarrow \pi^{ \pm} \pi^{0} \gamma$ decay can be conveniently described using the $T_{\pi}^{*}$, variables, where $T_{\pi}^{*}$ is the kinetic energy of the charged pion in the kaon rest frame and $W$ is 

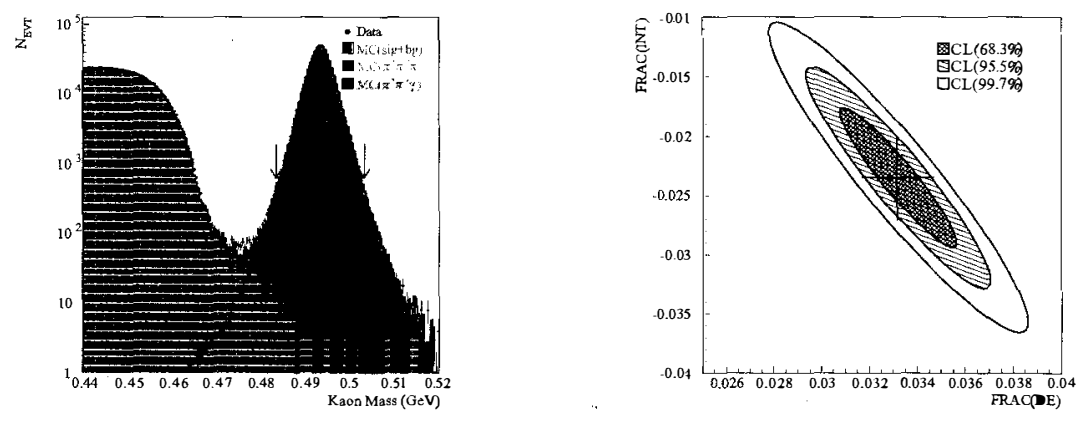

Figure 1: a) Kaon mass distribution for $K^{ \pm} \rightarrow \pi^{ \pm} \pi^{0} \gamma$ candidates b) Contour plot in the DE INT plane.

Lorentz invariant variable given by ${ }^{2,3}$ :

$$
W^{2}=\frac{\left(P_{K} \cdot P_{\gamma}\right)\left(P_{\pi} \cdot P_{\gamma}\right)}{\left(m_{K} m_{\pi}\right)^{2}}
$$

$P_{K}, P_{\pi}, P_{\gamma}$ are the 4 momenta of the kaon, the charged pion and the radiative gamma. Using these variables, the differential rate for the $K^{ \pm} \rightarrow \pi^{ \pm} \pi^{0} \gamma$ process can be written as ${ }^{2,3,6}$ :

$$
\begin{array}{r}
\frac{\partial^{2} \Gamma^{ \pm}}{\partial T_{\pi}^{*} \partial W}=\frac{\partial^{2} \Gamma_{I B}^{ \pm}}{\partial T_{\pi}^{*} \partial W}\left[1+2 \cos \left( \pm \phi+\delta_{1}^{1}-\delta_{0}^{2}\right) m_{\pi}^{2} m_{K}^{2}\left(\left|X_{E}\right|\right) W^{2}+\right. \\
\left.m_{\pi}^{4} m_{K}^{4}\left(\left|X_{E}\right|^{2}+\left|X_{M}\right|^{2}\right) W^{4}\right]
\end{array}
$$

where $\frac{\partial^{2} \Gamma_{I B}^{ \pm}}{\partial T_{\pi}^{*} \partial W}$ is the differential rate for the IB component, $\phi$ is the CP violating phase, $\delta_{L}^{I}$ are ;he strong $\pi-\pi$ re-scattering phases for a final state of momentum $L$ and isospin $I$, and $X_{E}, X_{M}$ tre normalized electric and magnetic amplitudes respectively.

In the NA48/2 analysis the main background (BG) sources are $K^{ \pm} \rightarrow \pi^{ \pm} \pi^{0}$ and $K^{ \pm} \rightarrow$ $\tau^{ \pm} \tau^{0} \pi^{0}$. The first decay needs an accidental photon or an hadronic extra cluster to mimic the ;ignal final state, while the second a lost or two fused $\gamma$. The rejection of $K^{ \pm} \rightarrow \pi^{ \pm} \pi^{0}$ relies on ihe $T_{\pi}^{*}<80 \mathrm{MeV}$ cut. To suppress $K^{ \pm} \rightarrow \pi^{ \pm} \pi^{0} \pi^{0}$ BG the very good kaon mass resolution, (2.2 $\mathrm{MeV}$ ) and the identification of fused gamma events by kinematical constraints have been used. $n$ fig. 1 the data kaon mass spectrum is compared with the sum of signal plus BG MC. To :orrectly reconstruct the $W$ value is very important to distinguish the radiative $\gamma$ from the two :oming from the $\pi r^{0}$ decay. Using a set of cuts a misidentification probability, computed using $\mathrm{CC}$ simulation, lower than the permille for all the components has been achieved. At the end of he selection $\sim 600 K K^{ \pm} \rightarrow \pi^{ \pm} \pi^{0} \gamma$ candidates have been identified with a BG contamination $=1 \%$ with respect to the DE component.

The extraction of DE and INT fractions relies on their different W distribution. An algorithm ased on extended maximum likelihood assigns weights to MC W distributions of the three omponents to reproduce data. The fit has been performed in the region $0.2<W<0.9$. The ollowing values for the DE and INT fractions have been obtained:

$$
\begin{aligned}
& \operatorname{Frac}(\mathrm{DE})_{\mathrm{T}_{\pi}^{*}<80 \mathrm{MeV}}=\left(3.32 \pm 0.15_{\text {sta }} \pm 0.14_{\text {sys }}\right) \times 10^{-2} \\
& \operatorname{Frac}(\mathrm{INT})_{\mathrm{T}_{\pi}^{*}<80 \mathrm{MeV}}=-\left(2.35 \pm 0.35_{\text {sta }} \pm 0.39_{\text {sys }}\right) \times 10^{-2}
\end{aligned}
$$

rith a $\chi^{2}$ of the residuals with respect to zero of $14.3 / 13$ d.o.f. This is the first measurement of non vanishing interference term in the $K^{ \pm} \rightarrow \pi^{ \pm} \tau^{0} \gamma^{\prime}$ decay. The contour plot in Fig. 1 shows 
the very high correlation $(-0.93)$ of the two contributions. In order to compare the NA48 result with those from previous experiments, the ML fit has been redone setting the INT te to zero. The $\chi^{2}$ of the residuals, 51/13 d.o.f., demonstrates that the data distribution cann be properly described without an INT term.

To investigate CP violation in $K^{ \pm} \rightarrow \pi^{ \pm} \pi^{0} \gamma$ with maximum statistical accuracy, the wh $W$ spectrum has been considered. Candidates were separated depending on the reconstruct kaon charge, leading to a sample with $695 \times 10^{3} \mathrm{~K}^{+}$and $386 \times 10^{3} \mathrm{~K}^{-}$. The simplest CP violati observable, the difference in the decay rates of $K^{+}$and $K^{-}$, can be expressed in term of numb of events as:

$$
A_{N}=\left(N_{+}-R N_{-}\right) /\left(N_{+}+R N_{-}\right)
$$

where $N_{+}, N_{-}$are the number of $K^{+}, K^{-}$decays to $\pi^{ \pm} \pi^{0} \gamma$, and $R$ is the ratio of the number $K^{+}$to $K^{-}$in the beam, computed from another channel for which no CP violation is expecte The NA48/2 data has shown no CP violation asymmetry in the Dalitz plot for $K^{ \pm} \rightarrow \pi^{ \pm} \pi^{0}$ decays at the level of $10^{-41}$. The large number of $K^{ \pm} \rightarrow \pi^{ \pm} \pi^{0} \pi^{0}$ decays collected, allows $t$ measurement of $R$ with an accuracy of $\delta R / R \sim 2 \times 10^{-4}$. The ratio $R$ has been computed bins of kaon momentum and the asymmetry $A_{N}$ calculated to be:

$$
A_{N}=\left(0.0 \pm 1.0_{s t a} \pm 0.6_{\text {sys }}\right) \times 10^{-3}
$$

From the above value a limit for the rate asymmetry of $\left|A_{N}\right|<1.5 \times 10^{-3}$ at $90 \%$ CL be deduced. Another interesting observable of $\mathrm{CP}$ violation can be obtained by fitting asymmetry distribution as function of the variable $W$. Using Equation 2, the following dependence of the asymmetry $\left(A_{W}\right)$ is predicted:

$$
\frac{d A_{W}}{d W}=\frac{e W^{2}}{1+a W^{2}+b W^{4}}
$$

The parameters $a$ and $b$ are related to the fractions of DE and INT, while $e$ is left free in $\mathrm{t}$ fit. Using the value of $e$ obtained from the fit $A_{W}$ is computed to be: $A_{W}=(-0.6 \pm 1.0) \times 10$ The value of both $A_{N}$ and $A_{W}$ are consistent with no charge asymmetry.

\section{The $K^{ \pm} \rightarrow \pi^{ \pm} e^{+} e^{-}$decay rate and form factor}

The flavour-changing neutral current process $K^{ \pm} \rightarrow \pi^{ \pm} e^{+} e^{-}$, induced at one-loop level in $t$ Standard Model and highly suppressed by the GIM mechanism, is of particular interest. T decay is supposed to proceed through one photon exchange, resulting in a spectrum of $\mathrm{t}$ $z=\left(M_{e e} / M_{K}\right)^{2}$ kinematic variable sensitive to the form factor $\mathrm{W}(\mathrm{z})$. In ChPT several mode predicting the form factor characterizing the dilepton invariant mass spectrum, and thus $t$ decay rate, have been proposed ${ }^{7,8}$. The following parameterizations of the form factor $\mathrm{W}(\mathrm{z})$ considered in the analysis:

1. Linear: $W(z)=G_{F} M_{K}^{2} f_{0}(1+\delta z)$

with free normalization and slope $\left(f_{0}, \delta\right)$.

2. Next-to-leading order ChPT: $\quad W(z)=G_{F} M_{K}^{2} f_{0}\left(a_{+}+b_{+} z\right)+W^{\pi \pi}(z)$ with free parameters $\left(a_{+}, b_{+}\right)$, and an explicitly calculated pion loop term $W^{\pi \pi}(z)^{7}$. 3. The Dubna version of ChPT involving meson form factors: $\quad W(z) \equiv W\left(M_{a}, M_{\rho}, z\right)$ with resonance masses $\left(M_{a}, M_{\rho}\right)$ treated as free parameters.

After Moriond conference we discovered the existence of a $4^{\text {th }}$ model for the $K^{ \pm} \rightarrow \pi^{ \pm} e^{+}$ form factor ${ }^{9}$. The result of the fit to all the four models can be found in reference ${ }^{10}$.

In NA48/2 analysis the $K^{ \pm} \rightarrow \pi^{ \pm} e^{+} e^{-}$rate is measured relatively to the abundant $K^{ \pm}$ $\pi^{ \pm} \pi_{D}^{0}$. The final states of the signal and normalization channels contain identical sets of charg particles. Thus particle identification efficiencies, representing a significant source of systema 

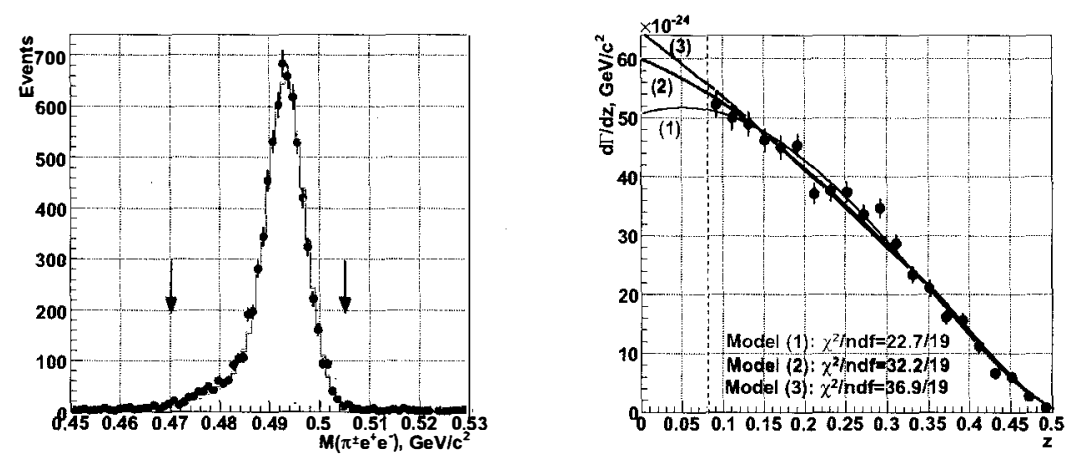

Figure 2: a) Reconstructed $\pi^{ \pm} e^{+} e^{-}$invariant mass: data (dots) and MC simulation (filled area) b) Computed $\mathrm{d} \Gamma_{e e} / \mathrm{dz}$ and fit results according to the threc considered models.

incertainties, cancel in the first order. At the end of the selection on whole 20032004 data ámple 7253 candidates $K^{ \pm} \rightarrow \pi^{ \pm} e^{+} e^{-}$are found in the signal region with $1.0 \%$ background ontamination (see Fig.2).

The result of fits to the distribution of rate vs $z$, using the three different parametrization ure shown in Fig.2. All the fits are of reasonable quality, however the linear form-factor model eads to the smallest $\chi^{2}$. Values of all form factor parameters can be found in reference ${ }^{10}$. Infortunately the data sample is insufficient to distinguish between the models considered. The branching ratio in the full kinematic range, which is computed as the average between he two extremes corresponding to the models (1) and (3), and includes an uncertainty due to $\mathrm{xtrap}$ lation into the inaccessible region $z<0.08$, is:

$$
B R_{M I}=\left(3.11 \pm 0.04_{\text {sta }} \pm 0.05_{\text {sys }} \pm 0.08_{\text {ext }} \pm 0.07_{\text {mod }}\right) \cdot 10^{-7}=(3.11 \pm 0.12) \cdot 10^{-7}
$$

inally, a first measurement of the direct CP violating asymmetry of $K^{+}$and $K^{-}$decay rates in he full kinematic range was obtained by performing BR measurements separately for $K^{+}$and $i^{-}$and neglecting the correlated uncertainties: $\Delta\left(K_{\pi e e}^{ \pm}\right)=\left(B R^{+}-B R^{-}\right) /\left(B R^{+}+B R^{-}\right)=$ $\left.-2.2 \pm 1.5_{\text {sta }} \pm 0.6_{\text {sys }}\right) \%$. A conservative limit for the charge asymmetry of $\left|\Delta\left(K^{ \pm} \rightarrow \pi^{ \pm} e^{+} e^{-}\right)\right|<$ $.1 \cdot 10^{-2}$ at $90 \% C L$ can be deduced. The result is consistent with no $\mathrm{CP}$ violation.

\section{leferences}

1. J. R. Batley et al. [NA48/2 Collaboration], Eur. Phys. J. C 52, 875 (2007)

2. N. Christ, Phys. Rev. 159, 1292 (1967)

3. G. D'Ambrosio, M. Miragliuolo and P. Santorelli, The Daфne Physics Handbook, Eds. L. Maiani, G. Pancheri and N. Paver (1992)

4. H. Y. Cheng, Phys. Rev. D 42, 72 (1990)

5. G. Ecker, H. Neufeld, A. Pich, Nucl. Phys. B 413, 321 (1994)

6. L. Cappiello and G. D'Ambrosio, Phys. Rev. D 75, 094014 (2007)

7. G. D’Ambrosio, G. Ecker, G. Isidori and J. Portoles, JHEP 9808, 004 (1998)

8. A. Z. Dubnickova, S. Dubnicka, E. Goudzovski, V. N. Pervushin and M. Secansky, arXiv:hep-ph/0611175.

9. S. Friot, D. Greynat and E. De Rafael, Phys. Lett. B 595, 301 (2004)

10. J. R. Batley et al. [NA48/2 Collaboration], arXiv:0903.3130 [hep-ex]. 